\title{
Free-breathing black-blood CINE fast-spin echo imaging for measuring abdominal aortic wall distensibility: A feasibility study
}

\author{
Jyh-Miin Lin ${ }^{1,2}$, Andrew J. Patterson ${ }^{3}$, Tzu-Cheng Chao ${ }^{4}$, Chengcheng \\ Zhu $^{5}$, Hing-Chiu Chang ${ }^{6}$, Jason Mendes ${ }^{7}$, Hsiao-Wen Chung ${ }^{2}$, \\ Jonathan H. Gillard ${ }^{1}$ and Martin J. Graves ${ }^{3}$ \\ ${ }^{1}$ Department of Radiology, University of Cambridge, Cambridge, CB2 0QQ, United Kingdom \\ ${ }^{2}$ Graduate Institute of Biomedical Electronics and Bioinformatics, National Taiwan University, \\ Taipei, Taiwan \\ ${ }^{3}$ MRIS Unit, Cambridge University Hospitals NHS Foundation Trust, Cambridge, CB2 0QQ, \\ United Kingdom \\ ${ }^{4}$ Department of Computer Science and Information Engineering, National Cheng Kung \\ University, Tainan, Taiwan \\ ${ }^{5}$ Department of Radiology, UCSF School of Medicine, San Francisco, California, USA \\ ${ }^{6}$ Department of Diagnostic Radiology, Li Ka Shing Faculty of Medicine, The University of Hong \\ Kong, Hong Kong \\ ${ }^{7}$ Department of Radiology, University of Utah, Salt Lake City, UT 84112, USA \\ Correspondence to: J-M. Lin \\ E-mail: jml86@cam.ac.uk and jyhmiinlin@gmail.com
}

\begin{abstract}
The paper reports a free-breathing black-blood CINE fast-spin echo (FSE) technique for measuring abdominal aortic wall motion. The free-breathing CINE FSE includes the following MR techniques: 1) variable-density sampling with fast iterative reconstruction; 2) inner-volume imaging; and 3) a blood-suppression preparation pulse. The proposed technique was evaluated in eight healthy subjects. The inner-volume imaging significantly reduced the intraluminal artifacts of respiratory motion $(\mathrm{p}=0.015)$. The quantitative measurements were a diameter of $16.3 \pm 2.8$ $\mathrm{mm}$ and wall distensibility of $2.0 \pm 0.4 \mathrm{~mm}(12.5 \pm 3.4 \%)$ and $0.7 \pm 0.3 \mathrm{~mm}(4.1 \pm 1.0 \%)$ for the anterior and posterior walls, respectively. The cyclic cross-sectional distensibility was $35 \pm 15 \%$ greater in the systolic phase than in the diastolic phase. In conclusion, we developed a feasible CINE FSE method to measure the motion of the abdominal aortic wall, which will enable clinical scientists to study the elasticity of the abdominal aorta.
\end{abstract}

Keywords: CINE FSE; abdominal aortic wall distensibility; local excitation; inner-volume imaging; iterative image reconstruction; parallel computing

Submitted to: Phys. Med. Biol. 


\section{Introduction}

Aortic distensibility is increasingly considered a potential prognostic parameter of cardiovascular disease (Nelson et al , 2009; Paraskevas et al , 2009; Redheuil, 2014; Soljanlahti et al , 2008). Reduced elasticity is observed in patients (Baumgartner et al , 2006; Hickson et al , 2010; Seo et al , 2009; Tsamis et al , 2013; Voges et al , 2012) as a result of abdominal aortic aneurysms (Abbas et al , 2015; Hall et al , 2000), hypertension, smoking (Ohyama et al , 2016), ageing (Langham et al , 2015; Sakuragi and Abhayaratna, 2010; Taviani et al , 2011), and diastolic heart failure (Hundley et al , 2001). Non-contrast enhanced MRI plays a versatile role in aortic wall imaging, since brightblood and black-blood MR pulse sequences together provide mutually complementary information. While bright-blood imaging excels in indicating the intraluminal pathology and abnormal blood flows, the black-blood spin echo sequence is the standard MR sequence for evaluating pathological changes in the aortic walls, such as intramural hematoma (Nienaber et al , 2009), wall thickening, and aortic dissection (Donati et al , 2015; Nienaber and Powell, 2011).

Time-resolved CINE imaging acquires images at multiple time points to depict the aortic wall dynamics over the cardiac cycle. Current available CINE imaging relies on bright-blood fast gradient echo sequence, with a short repetition time (TR), to enable multiple cardiac phases to be efficiently acquired. Bright-blood CINE imaging clearly demonstrates the intraluminal blood flow, revealing the aortic wall by a negative contrast. In bright-blood CINE imaging, a dark area surrounding the lumen is identified as the aortic wall. For quantitative measurements, however, this negative contrast of the aortic wall can be influenced by pulsatile fluctuations and turbulence in blood flows. The alternative black-blood CINE imaging can directly visualize the aortic wall because intramural pathological changes can be identified without the partial volume effects from intraluminal flow signal. Black blood CINE imaging was achieved through the use of customized ECG-gated gradient echo sequences with spatial saturation pulses (Taviani et al , 2011); however, the boundary definition can be poor in diastole.

CINE FSE of the abdominal aorta may be less susceptible to inaccuracies due to pulsatile fluctuations and turbulence in blood flows. Unlike bright-blood CINE imaging, however, blackblood CINE FSE is not readily available as a common clinical sequence for measuring the aortic wall motion. Two problems related to dual triggered CINE FSE have been recognized. First, CINE FSE with the constant long TR is less efficient than the short TR CINE gradient echo sequence when multiple cardiac phases must be acquired. It is inefficient to acquire a targeted cardiac phase in coincidence with the fixed TR interval. Thus, ECG-triggered CINE FSE is slower than CINE gradient echo sequences for time-resolve CINE imaging. Second, respiratory motion artifacts pose another challenge because prospective triggering leads to varying TR intervals. Retrospective respiratory gating faces a similar problem related to long TR.

This paper describes the development of a free-breathing black-blood CINE FSE method for measuring abdominal aortic wall motion. Recently, CINE FSE sequences have combined variabledensity sampling with advanced reconstruction methods (Boesen et al , 2015; Mendes et al , 2011) to perform black blood CINE imaging of the carotid artery. This study implemented a similar variabledensity sampling strategy (Boesen et al , 2015; Mendes et al , 2011) to allow efficient acquisitions, together with inner volume excitation (Feinberg et al, 1985) and improved blood suppression to allow free-breathing black-blood CINE FSE of the abdominal aorta. In addition, reconstruction speed was improved through parallel computing. The distensibility of the abdominal aortic wall was obtained from the black-blood CINE FSE sequence. The free-breathing CINE FSE sequence was also compared with the standard bright-blood CINE balanced steady state free precession (bSSFP) 
and CINE RF-spoiled gradient echo sequences.

\section{Materials and Methods}

The schematic diagram of the free-breathing CINE FSE is shown as Figure 1. Each component is detailed in the following sections.

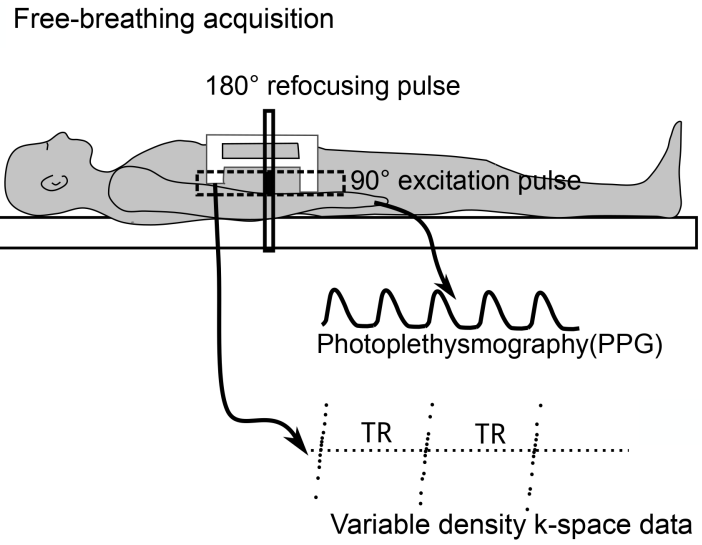

Figure 1.

Schematic diagram of the free-breathing CINE fast-spin echo (FSE) for measuring abdominal aortic wall motion. Photoplethysmography (PPG) and MRI raw data are concurrently acquired. Raw data are reordered according to the PPG (See Figure 4). After iterative reconstruction, the crosssectional area changes between the systolic and diastolic phases are measured (Figure 6).

\subsection{Pulse sequence}

Free-breathing CINE FSE is illustrated in Figure 2. An FSE sequence was modified to include: 1) delay alternating with nutation for tailored excitation (DANTE) (Li et al , 2012) pre-pulse for flow suppression, 2) inner-volume excitation, and 3) variable-density sampling.

The DANTE pre-pulse employs multiple small angle excitation and dephasing gradients with a short time delay (Td) of $100 \mathrm{~ms}$. Each DANTE pre-pulse was added before the $90^{\circ}$ excitation pulse. Lipid suppression is optional: when lipid suppression is turned on, an inversion pulse is added behind DANTE and ahead of the $90^{\circ}$ excitation pulse.

A $90^{\circ}$ Shinnar-Le Roux (SLR) radio frequency pulse (Pauly et al , 1991) with less than $0.1 \%$ ripple in the pass band and stop band was employed to generate an orthogonal excitation plane (Figure 3). The duration of the SLR pulse was restricted to $3.2 \mathrm{~ms}$ to maintain the original interecho spacing. The width of the SLR excitation region was controlled by the gradient strength (Gy in Figure 2). Banding artifacts from the DANTE and SLR pulses were eliminated by crusher gradients.

Reduced FOV acquisition has been accepted as an effective method of suppressing the residual respiratory artifacts in thoracic and abdominal MRI. Although there are subtle superior-inferior movements in the descending aorta (Clough et al , 2011), we found that such minor aortic 
(A)

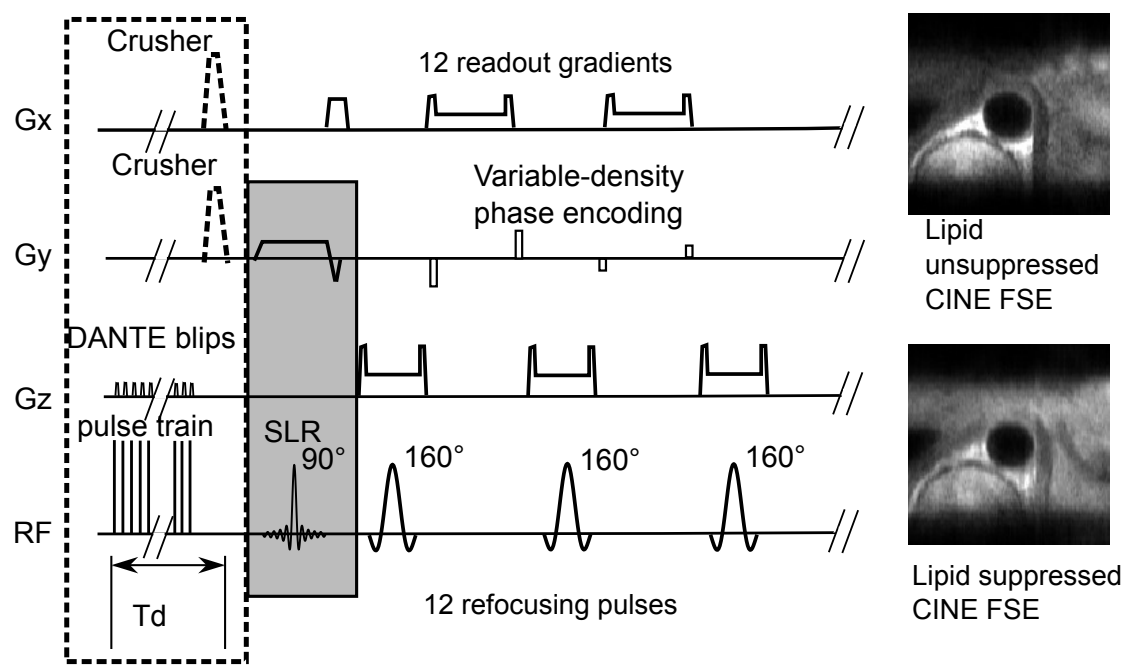

(B)

Figure 2.

(A) Sequence diagram for free-breathing, inner-volume CINE FSE, including the DANTE pre-pulse, inner-volume excitation (90 ${ }^{\circ} \mathrm{SLR}$ pulse) and variable-density acquisition. The preparation time for the DANTE (Td) was $100 \mathrm{~ms}$. (B) Lipid unsuppressed CINE FSE (upper) and lipid suppressed CINE FSE (lower). If lipid suppression is turned on, an inversion pulse is added between DANTE and $90^{\circ}$ SLR pulse.

movements rarely cause visible respiratory motion artifacts. In other words, the abdominal aorta is effectively static and is free of motion artifacts.

Variable-density sampling changes the phase encoding by using a predetermined view table. As the sampling density is uneven (see Figure 4(B)), the view table is randomized.

\subsection{Retrospective photoplethysmography (PPG) gating}

Figure 4 illustrates the concept of variable-density sampling, which retrospectively constructs the sparse k-space domain and time domain $\left(k_{y}-t\right)$ for the subsequent image reconstruction. The variable-density sampling method based on Mendes et al. (Mendes et al , 2011) was already established, and we doubled the number of excitations to 88 to increase the signal-to-noise ratio (SNR) around the abdominal aorta. The sampling density is higher at the center of the k-space than the peripheral k-space: the center of the k-space has 24 temporal samples, and there are 96 samples distributed across the -2 and +2 lines. The density of center of $\mathrm{k}$-space is sufficient for the reconstruction of 16 cardiac phases.

The PPG waveform was simultaneously acquired from the middle finger of each subject. At the end of acquisition, the acquired raw data were correlated with the PPG waveform data, and each phase encoding was given a value of $t$, which stands for the cardiac phase. This sparse sampling 

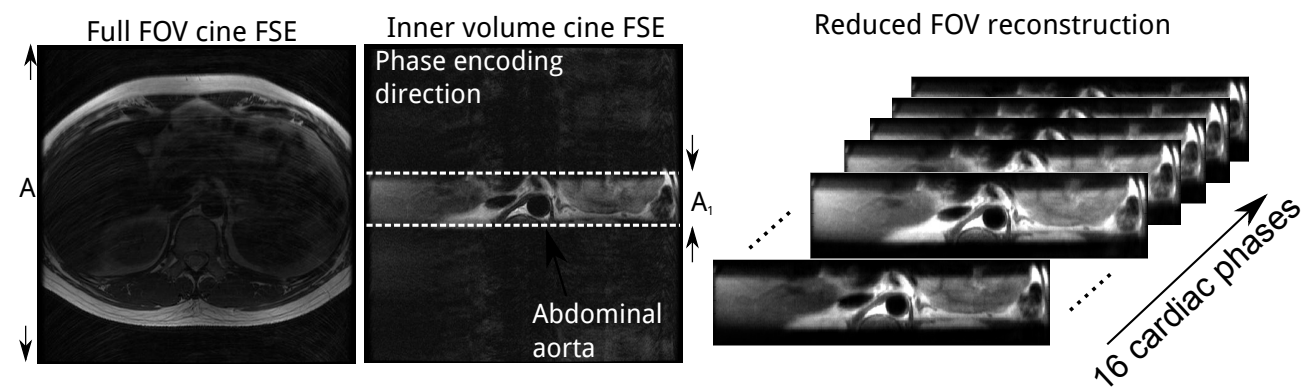

\section{Figure 3.}

Elimination of respiratory motion artifacts using the proposed inner-volume CINE FSE. The innervolume excitation excludes the respiratory motion from the anterior abdominal wall. Therefore, the abdominal aortic wall motion can be imaged using the CINE FSE method. Reduced FOV reconstruction saves the computation time.
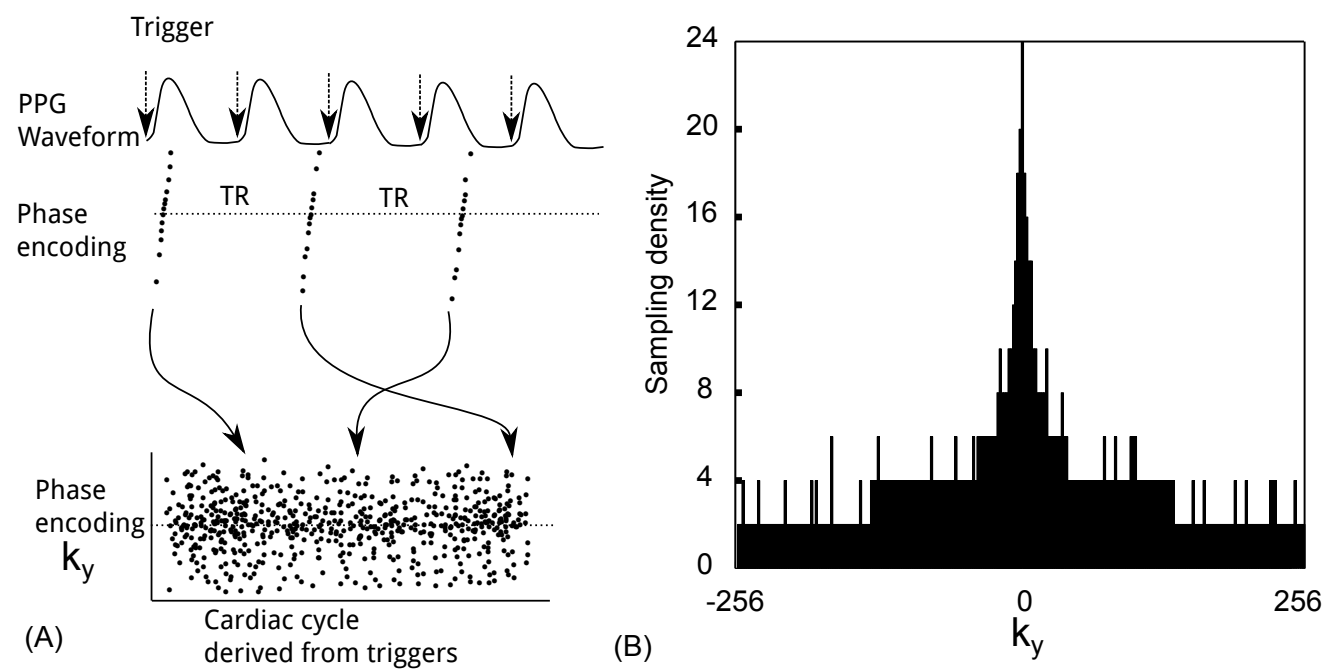

Figure 4.

Data reordering and variable-density sampling. (A) Retrospective PPG gating implemented in CINE FSE. The raw data were correlated with the PPG data, generating a sparse $k_{y}-t$ space with variable density. This technique does not require prospective cardiac gating. Instead, each $\mathrm{k}$-line was given a temporal value $\mathrm{t}$ according to its position in relation to the cardiac cycle. The dotted line indicates the center of the phase encoding. (B) The variable-density phase encodings $\left(k_{y}\right)$ provide sufficient temporal resolution and efficient acquisition.

scheme in the $k_{y}-t$ domain is equivalent to an acceleration factor of 3.88 . 


\subsection{Reconstruction of sparse data}

The undersampled $k_{y}-t$ space induces abrupt variations along the spatial and temporal axes, which could be eliminated by enforcing spatial and temporal smoothness (called regularizations). However, a routine FFT does not allow for spatial and temporal regularizations, and an iterative algorithm is needed for regularization purposes. The implemented reconstruction was based on the method proposed by Mendes et al. (Mendes et al , 2011) and the temporally constrained reconstruction (Adluru et al , 2007). A slight difference between previous work and this study is that we added the spatial regularization terms. The iterative conjugate gradient reconstruction algorithm solves the following equation:

$$
\begin{aligned}
m= & \operatorname{argmin}_{a}\left(\left\|R F m(x, y, t)-D\left(k_{x}, k_{y}, t\right)\right\|_{2}^{2}+\right. \\
& \left.\lambda_{t}\left\|\nabla_{t} m(x, y, t)\right\|_{2}^{2}+\lambda_{x}\left\|\nabla_{x} m(x, y, t)\right\|_{2}^{2}+\lambda_{y}\left\|\nabla_{y} m(x, y, t)\right\|_{2}^{2}\right)
\end{aligned}
$$

where $m(x, y, t)$ is the result of temporal frames in CINE FSE, $D$ is the raw data acquired in the $k_{x}-k_{y}-t$ space, $F$ is the imaging encoding that maps CINE images $m(x, y, t)$ to the $k_{x}-k_{y}-t$ space, and $R$ is the variable-density sampling pattern retrospectively obtained from the PPG. Note that in Equation 1 coil sensitivities have been incorporated into the encoding matrix. The partial derivative $\nabla_{t}$ is the temporal gradient. The temporal regularization is weighted by constant $\lambda_{t} . \nabla_{x}$ and $\nabla_{y}$ are image gradients along the $\mathrm{x}$ and $\mathrm{y}$ directions and are weighted by $\lambda_{x}$ and $\lambda_{y}$. To limit temporal blurring, we designated $\lambda_{t}=0.1$, and $\lambda_{x}=\lambda_{y}=0.003$. Although the temporal constraint in this study was a constant that was lower than the values provided in Mendes et al. (17), the current reconstruction was modified for sufficient temporal resolution and SNR, making the images appropriate for the subsequent measurements.

The typical conjugate gradient method uses the following gradient term during iterations:

$$
\begin{aligned}
G(m)= & F^{T} R^{T}\left(R F m(x, y, t)-D\left(k_{x}, k_{y}, t\right)\right)- \\
& \lambda_{t} \Delta_{t} m(x, y, t)-\lambda_{x} \Delta_{x} m(x, y, t)-\lambda_{y} \Delta_{y} m(x, y, t)
\end{aligned}
$$

where $\Delta_{t}, \Delta_{x}$, and $\Delta_{y}$ indicate the discrete Laplacian operators. Here, the spatio-temporal Laplacian was implemented by multiplying the $k-f$ data by the modulation kernel, which is the 3D fast Fourier transform (FFT) of the standard 3D discrete Laplacian operator. Note that Equation 2 can be applied to the inner-volume CINE FSE with an rFOV.

Figure 5 validates the effects of the iterative algorithm with spatial and temporal regularizations. The results after the 1 st and 60 th iterations were compared with the final result after 200 iterations. The difference images of the 60 th iteration show fewer artifacts than the 1st iteration.

\subsection{Accelerated reconstruction}

One issue associated with iterative image reconstruction is the long run time. We implemented a fast algorithm, which is one order of magnitude faster than the generic version. See the Appendix for details of the accelerated implementation.

\subsection{In vivo imaging}

Eight healthy subjects ( 4 males and 4 females; $30 \pm 6$ years old) provided written informed consent for this ethically approved technical study. The experiments were conducted using a $1.5 \mathrm{~T}$ scanner 
(A)

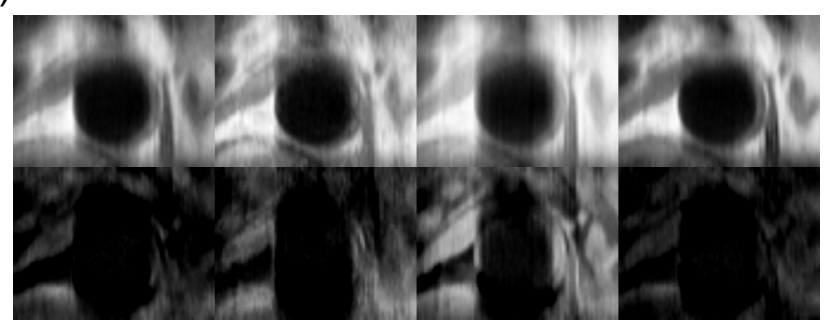

(B)

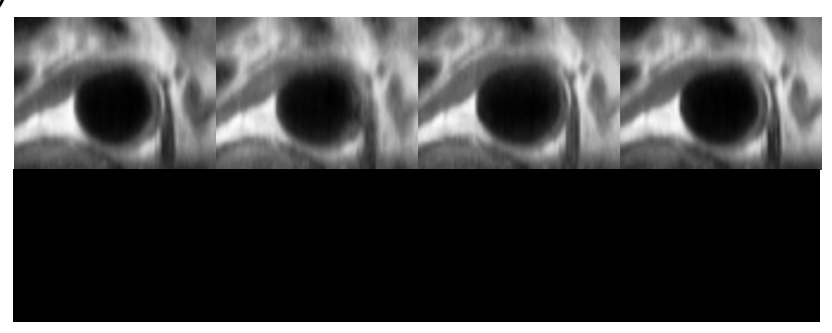

Figure 5.

The convergence of the implemented reconstruction (A) 4 images selected from 16 cardiac phases after the 1st FFT (upper row) and images of the difference (lower row) between the 1st and the 200th iterations; (B) 4 images selected from 16 cardiac phases after the 60th iterations (upper row) and images of the difference (lower row) between the 60 th and the 200th iterations. The undersampling artifact, due to sparse $k_{y}-t$ space, is eliminated after a certain number of iterations.

(MR450, GE Healthcare) and a 3T scanner (MR750, GE Healthcare) using 8-channel cardiac arrays. The scanning parameters were $\mathrm{TR}=2500 \mathrm{~ms}$ (therefore, the total scan time was $88 \times \mathrm{TR}=3: 40$ ); effective $\mathrm{TE}=51 \mathrm{~ms}$; echo train length $=12 ; \mathrm{FOV}=28 \mathrm{~cm} \times 28 \mathrm{~cm}$; matrix size $=512 \times 256$; and inter-echo spacing $=7.8 \mathrm{~ms}$ for $1.5 \mathrm{~T}$ and $9.5 \mathrm{~ms}$ for $3 \mathrm{~T}$.

Transverse CINE FSE images were obtained for the abdominal aorta at the level of the kidney. The simple superior-inferior pattern of motion during respiration was previously validated with subjects lying supine. Therefore, $15 \%$ of the phase FOV was sufficient to eliminate the respiratory artifacts from the abdominal wall.

The in vivo study compares the full FOV CINE FSE and inner-volume CINE FSE. As illustrated in Figure 2, two sequences only differ in the excitation methods. Both the DANTE pre-pulse and variable density sampling were kept identical.

\subsection{Comparison of free-breathing CINE FSE and bright-blood CINE MRI}

The free-breathing CINE FSE was compared with two bright-blood CINE MRI sequences: (a) breath-hold CINE fast imaging employing steady-state acquisition (FIESTA, GE Healthcare), and (b) the dual-trigger flow-compensated CINE RF-spoiled gradient recalled (SPGR) echo. To allow fair comparison between the three sequences, respiratory control in bright-blood CINE MRI was performed and the slice locations were identical to CINE FSE. Three participants aged $22 \pm 2$ years without cardiovascular disease or smoking history were scanned on a 3T scanner (Discovery MR750, 
GE Healthcare) using an 8-channel cardiac array. The CINE FIESTA data with unsuccessful breathhold were discarded. The parameters for CINE FIESTA were: TR: $3.978 \mathrm{~ms}$; TE: $1.732 \mathrm{~ms}$; matrix size: $256 \times 256$; flip angle: $35^{\circ}$; slice thickness: $5 \mathrm{~mm} ; 16$ cardiac phases; the total acquisition time of 4 slice positions was 8 minutes. Breath-hold CINE FIESTA required the subjects to hold their breath for 20 seconds and then rest until the pulse rate returned to normal before the next slice position was scanned. Free-breathing CINE SPGR has the following parameters: TR: 5.868 ms; TE: 2.9 ms; matrix size: $256 \times 256$, flip angle: $50^{\circ}$; 16 cardiac phases; slice thickness: $5 \mathrm{~mm}$. Respiratory triggered CINE SPGR allows free-breathing acquisitions; the respiratory triggering window was $20 \%$ at the end of expiration.

\subsection{Image evaluation}

Both the CINE FSE and the inner-volume CINE FSE used the DANTE blood suppression pulse. Therefore, the reduced intraluminal signal can be attributed to the reduction of respiratory motion artifacts. Because respiratory motion artifacts temporally fluctuate along the phase (y) direction (the anterior-posterior, AP axis), the intensity of intraluminal signal was measured from the $y-t$ plane (similar to M-mode image of ultrasound). The signal intensity within $80 \%$ of the intraluminal area was measured. To compare the different residual intraluminal signals between inner-volume and full FOV CINE FSE, non-parametric Wilcoxon signed-rank test (MATLAB version 2014a, The MathWorks, Inc., Natick, MA, USA) was used.

\subsection{Distensibility of the abdominal aortic wall}

The quantitative measurements of the abdominal aortic distensibility were obtained from the improved inner-volume CINE FSE. The wall motion was defined by the anterior-posterior between the peak systolic phase and the diastolic position. The cyclic changes of the cross-sectional area were manually quantified using a customized MATLAB program (Taviani et al , 2010). As can be seen in Figure 6, the area is plotted by smoothing a line passing through multiple nodes selected from the lumen-intima boundary. The maximum or minimum cross-sectional areas across the 16 cardiac phases are designated as the peaked systolic or diastolic phase, respectively.

\section{Results}

As shown in Figure 7(A), the full FOV CINE FSE had severe respiratory motion artifacts that overlaid the CINE images. The inner-volume CINE FSE method reduced such respiratory motion artifacts and improved the visualization of the aortic walls. This improvement was further confirmed by the statistical results (Figure 7(B)). The inner-volume CINE FSE method eliminated $66 \%$ of intraluminal artifacts, which significantly improved the image quality $(\mathrm{p}=0.015)$. The multiframe animation of Figure 7(A) is available at https://github.com/jyhmiinlin/cineFSE and in the Supplemental Material on the publisher's website.

One advantage of inner-volume CINE FSE is its accelerated reconstruction speed. Two reconstruction FOVs resulted in visually identical aortic cross-sections in the static transverse images (Figure 8(A)). The M-mode images shown in Figure 8(B) confirmed similar results between these two reconstruction methods. 
(A)
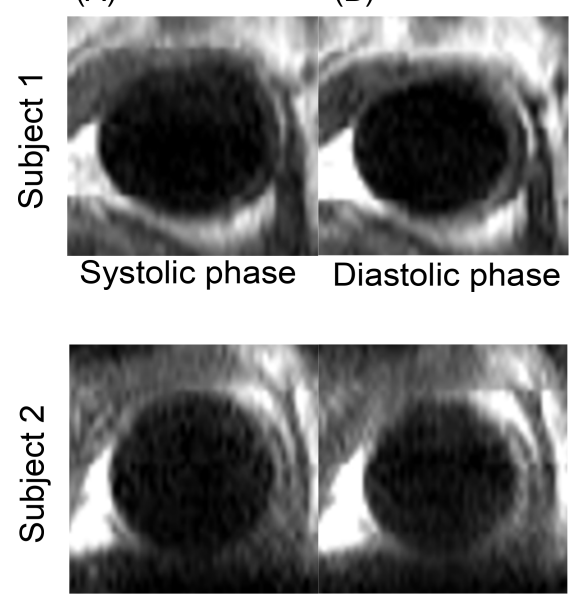

Systolic phase Diastolic phase
(C)

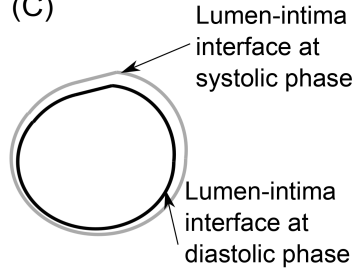

Area change

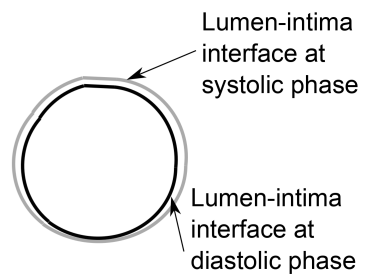

Area change

Figure 6.

Cross-sectional area changes of two subjects. (A) The light grey line illustrates the lumen-intima interface at the systolic phase. (B) The black line illustrates the lumen-intima interface at the diastolic phase. (C) The differences between area (A) and (B) divided by (B) indicate the area change in percentage. Peak systolic and diastolic phases are defined by the maximum and minimum cross-sectional area across the cardiac cycle (see Figure $9(\mathrm{~A})$ ).

(A)

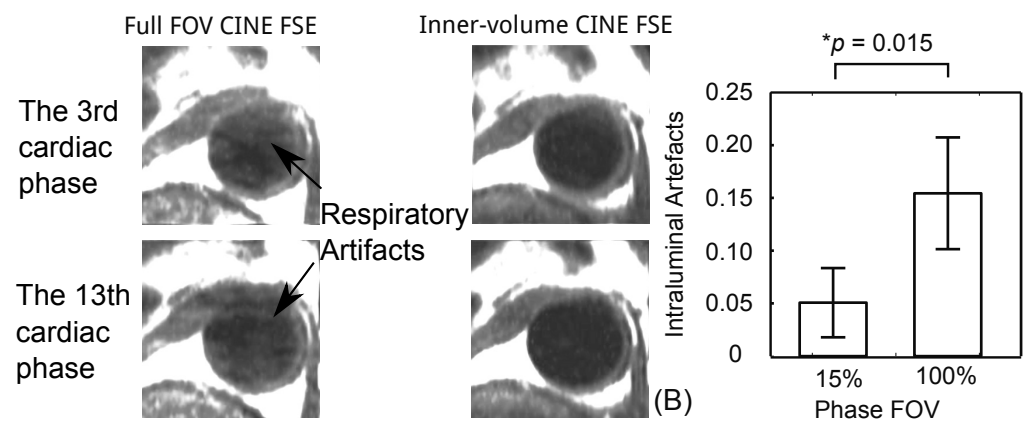

Figure 7.

(A) The two cardiac phases of the abdominal aorta. Apparent respiratory motion artifacts from the outer volume can be clearly observed in the full FOV CINE FSE (arrows), while the inner-volume CINE FSE has no such artifacts. The inner-volume CINE FSE shows superior image quality and fewer respiratory motion artifacts inside the lumen of the abdominal aorta. (B) The statistical comparison between the full FOV CINE FSE and the inner-volume CINE FSE shows that the latter eliminated $66 \%$ of the intraluminal artifacts. 
(A)

(B)

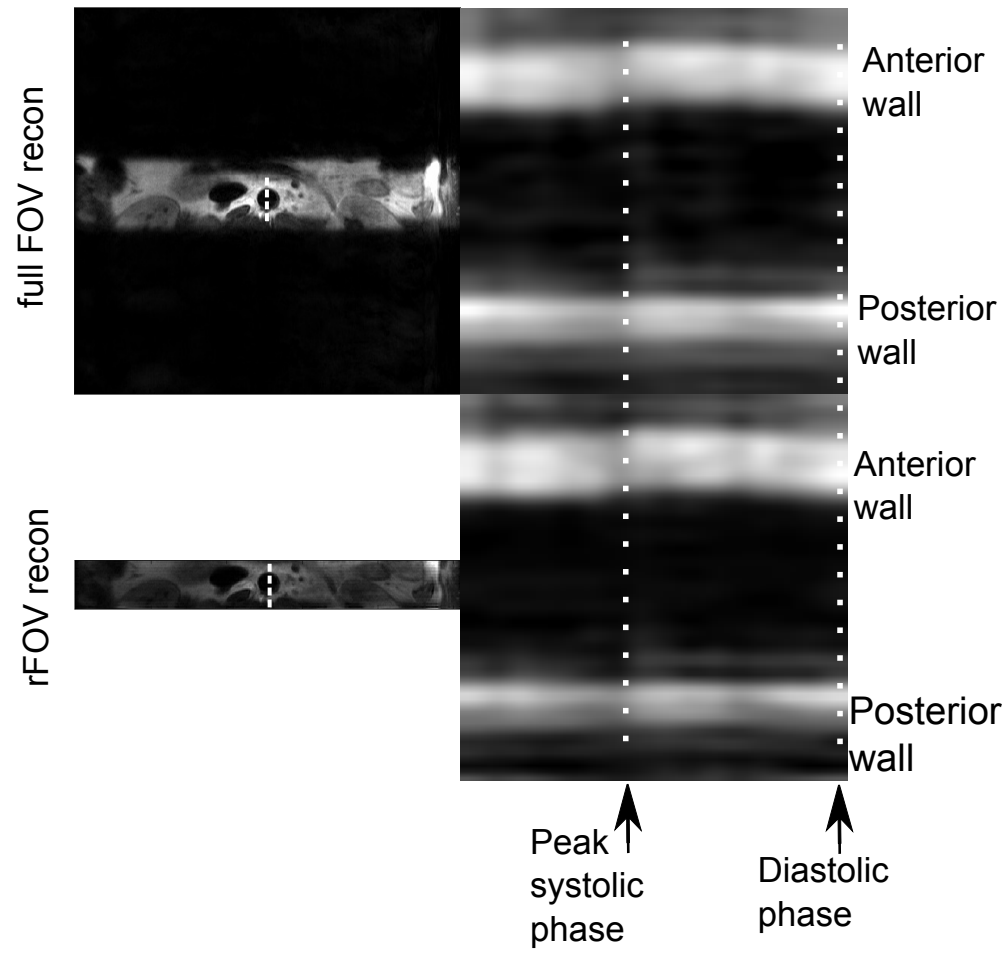

Cardiac Phases

Figure 8.

Comparison of the unaccelerated, full FOV reconstruction (full FOV recon) method and the accelerated reduced FOV reconstruction (rFOV recon) method. (A) The transverse slice. (B) The 16 temporal cardiac phases selected from the anterior/posterior line.

The mean diameter of the abdominal aorta for the eight volunteers was $16.3 \pm 2.8 \mathrm{~mm}$, which is within the normal range reported for healthy subjects (Morrison et al , 2009). As shown in Figure $9(\mathrm{~A})$, the distensibility of the cross-section area was $35 \pm 15 \%$ greater in the systolic phase than in the diastolic phase; this change is slightly higher than the previous value for healthy subjects (30.3\% reported in (Adams et al , 1995)). Figure 9(B) shows the aortic wall distensibility of 2.0 $\pm 0.4 \mathrm{~mm}(12.5 \pm 3.4 \%)$ and $0.7 \pm 0.3 \mathrm{~mm}(4.1 \pm 1.0 \%)$ for the anterior and posterior aortic walls, respectively. The anterior wall displacement was $3.3 \pm 1.2$ times greater than the posterior wall displacement, which is in agreement with the range of 2.47 to 4.03 previously reported in the literature (Goergen et al , 2007).

Figure 10 compares the black-blood CINE FSE with bright-blood CINE FIESTA and brightblood CINE SPGR. The healthy subject can breath-hold during CINE FIESTA and respiratory triggering is successful in CINE SPGR. The imaging plan is slightly inferior to celiac trunk, and 
bright-blood CINE MRI is sensitive to the turbulent flows at the systolic phase. Black-blood CINE FSE reduces the flow signals and is immune from such systolic turbulent flows. The animations for these three images can be viewed in the Supplemental Material on the publisher's website.

\section{Discussion}

We have developed a free-breathing black-blood CINE FSE which is insensitive to fluctuations in blood flow and respiratory motion artifacts. This technique may be useful when examining subjects with diminished breath-holding capability. Previous dual-triggered black-blood CINE FSE faces such problems as prolonged scanning times and irregular respiratory patterns with a variable acquisition window (Zho et al , 2010). Breath-hold is not required for CINE FIESTA of the aorta when the phase encoding direction is left-right. However, CINE FSE is sensitive to respiratory motion artifacts, and a free-breathing CINE FSE seems to be immune from issues related to respiratory triggering or repeated breath-hold.

The currently available technique of ECG-gated, fully sampled CINE FSE would take a scanning time approximately 16 times longer than static FSE. Unlike fully sampled CINE FSE, variable-density sampling can be performed within a scanning time only $2-4$ times longer than static FSE imaging (Mendes et al , 2011). This scanning time was tolerable for all the healthy subjects in this study. Results from healthy subjects showed that free-breathing CINE FSE can measure the aortic wall motion even when the subjects were allowed to breathe normally. Given that dual triggering may not be successful for patients who exhibit irregular respiratory patterns, free-breathing black-blood CINE MRI is a useful technique for measuring aortic wall motion in these clinical conditions.

The measured aortic distensibility aligned closely with the values reported in the literature (Adams et al , 1995; Goergen et al , 2007). This study confirmed the asymmetrical distensibility of aortic wall motions in healthy subjects, which was observed in an ultrasound study (Goergen et $a l, 2007$ ) and an ECG-gated, bright-blood CINE balanced fast gradient echo study (Van Prehn et
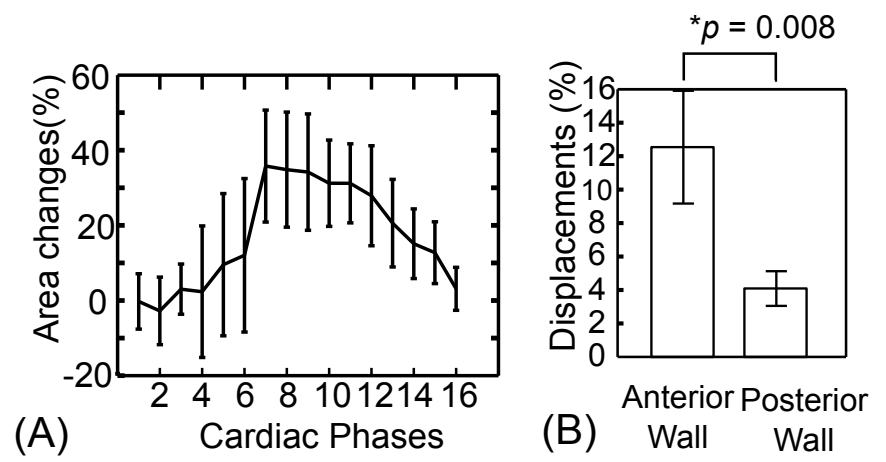

Figure 9.

(A) Cyclic area changes. The cross-sectional area of the systolic phase was $35 \pm 15 \%$ greater than that of the diastolic phase. (B) Asymmetrical differences in aortic wall motion. The anterior wall motion was greater than that of the posterior wall by a factor of 3.3. The difference between the anterior and posterior walls was statistically significant $(\mathrm{p}=0.008)$. 
(A) CINE FSE

(B) CINE bSSFP

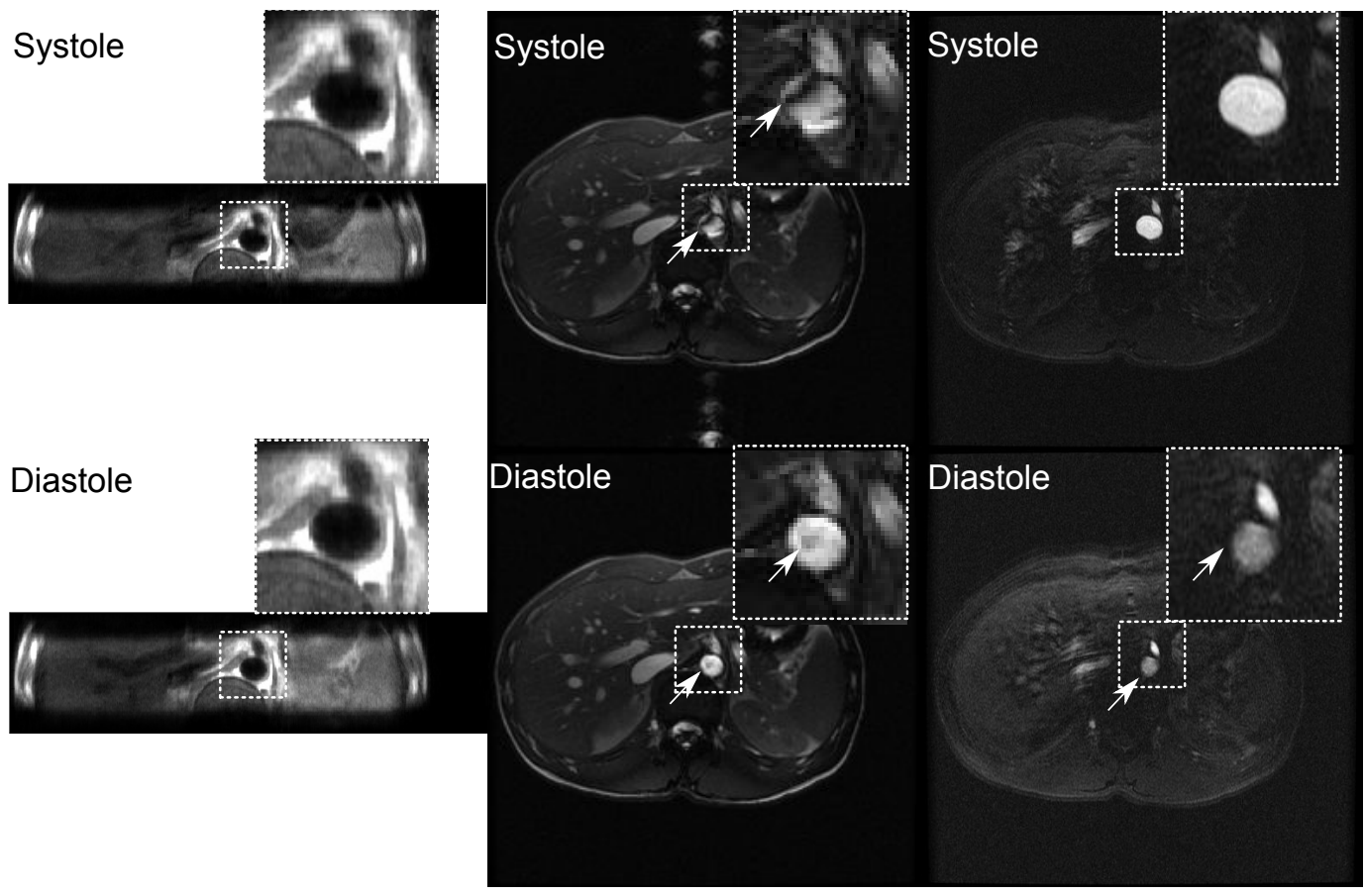

Figure 10.

(A) Free-breathing, black-blood CINE FSE was compared with (B) bright-blood CINE FIESTA with breath-hold, and (C) bright-blood CINE SPGR with respiratory triggering. The white arrows show low signal areas in the abdominal aorta, probably due to turbulent flows near the celiac trunk. Dual triggering may still suffer from motion related artifact from incoherent/inconsistent respiratory motion. Animations can be seen in Supplemental Material on the publisher's website.

$a l, 2009)$. In addition, the cyclic changes of aortic cross-sectional area were also consistent with the values previously revealed by ECG-triggered CT (Ganten et al , 2008).

Several potential improvements may increase the time efficiency of the free-breathing CINE FSE technique. However, our proposed free-breathing concept and reconstruction technique are still applicable to these improved techniques. Multi-slice inner volume excitation (Wargo et al , 2013) would be able to acquire the aortic wall motion of 6 - 12 slices in 3 minutes 40 seconds. It is also possible to further optimize the spatial resolution or acquisition times of the free-breathing CINE FSE technique, but at the expense of lower SNR. Beyond the Cartesian k-space with variabledensity sampling, non-Cartesian radial k-space is a more robust option for free-breathing self-gating segmented gradient echo acquisitions (Larson et al , 2004); still, three practical issues specific to radial CINE FSE are yet to be resolved. First, radial CINE FSE is not available on the current MRI scanners because variable T2 contrast and T2 reweighting influence the image quality (Altbach et al , 2002). Second, radial CINE FSE including the temporal axis is more computationally demanding and requires the non-uniform fast Fourier transform (NUFFT). Third, across a cardiac cycle, the blood signal of CINE FSE remains constantly black, which may not be ideal for the self-gating 
technique.

The diagonal dominant matrix in Equation 2 shows a practical way to partition a single problem into multiple smaller subproblems. If data are properly partitioned, this reduced FOV reconstruction also allows for parallel computing, which may further reduce the total computation time. Depending on the anatomical location of the organ, the $k_{y}-t$ sparse acquisition/reconstruction can be applied to a reduced FOV without the long computation time needed for full FOV reconstruction. A similar rFOV reconstruction has recently been proposed to accelerate iterative non-Cartesian reconstructions (Lin et al, 2015).

\section{Conclusions}

The free-breathing CINE FSE is a feasible method for measuring abdominal aortic wall motion. The measurements of the abdominal aortic wall motion are in agreement with previously published values.

\section{Appendix}

Accelerated reconstruction using parallel computing

Figure 3 illustrates the reduced size of volume A1 for iterative conjugate gradient reconstruction. Due to the smaller size of A1, inner-volume CINE FSE can be reconstructed more quickly than full FOV CINE FSE. The iterative conjugate gradient method repeats the FFT, which can be accelerated by the reduced FOV.

To further accelerate the reconstruction, parallel computing was implemented, and the data were decomposed into smaller partitions in the readout direction. This approach also simplified the software implementation because a Python object was generated for all sub-problems. Different sub-problems were distributed to multiple cores of the central processing unit (CPU). The different partitions were then integrated in the readout direction.

The image reconstruction algorithm was implemented in the Python programming language and deployed on a Linux workstation equipped with dual 10-core CPUs (Inte Xeon CPU E5-2650 v3, Intel, Santa Clara, CA, USA, clocked at $2.3 \mathrm{GHz}-3.0 \mathrm{GHz}$ (turbo-boost mode)) with $128 \mathrm{~GB}$ of memory. The reconstruction time was measured by the embedded time module. The acceleration was plotted against the number of parallel processes. The reconstruction program and examples are available in a public Github repository (https://github.com/jyhmiinlin/cineFSE).

The inner-volume CINE FSE accelerated the reconstruction by a factor of 2.79. Parallel computing further accelerated the reconstruction speed, and the combination of a reduced FOV and parallel computing achieved an acceleration factor of 23.26 .

\section{Acknowledgments}

The study was supported by Addenbrooke's Charitable Trust and the NIHR Comprehensive Biomedical Research Centre award to Cambridge University Hospitals NHS Foundation Trust, in partnership with the University of Cambridge. J.M.L. and H.W.C. received support from the Ministry of Science and Technology under grant Ministry of Science and Technology (MOST) grant: 
105-2221-E-002-142-MY3. T.C.C. and the Mind Research and Imaging Center at National Cheng Kung University acknowledge the financial support from MOST grant: 105-2314-B-006-044-MY2.

\section{References}

Abbas A, Cecelja M, Hussain T, Greil G, Modarai B, Waltham M, Chowienczyk P J and Smith A 2015 Thoracic but not abdominal phase contrast magnetic resonance-derived aortic pulse wave velocity is elevated in patients with abdominal aortic aneurysm J. Hypertens. 33(5), 1032-1038.

Adams J, Brooks M, Redpath T, Smith F, Dean J, Gray J, Walton S and Trent R 1995 Aortic distensibility and stiffness index measured by magnetic resonance imaging in patients with marfan's syndrome. Brit. Heart J. 73(3), 265-269.

Adluru G, Awate S P, Tasdizen T, Whitaker R T and DiBella E V 2007 Temporally constrained reconstruction of dynamic cardiac perfusion MRI Magn. Reson. Med. 57(6), 1027-1036.

Altbach M I, Outwater E K, Trouard T P, Krupinski E A, Theilmann R J, Stopeck A T, Kono M and Gmitro A F 2002 Radial fast spin-echo method for T2-weighted imaging and T2 mapping of the liver J. Magn. Reson. Imaging 16(2), 179-189.

Baumgartner D, Baumgartner C, Schermer E, Engl G, Schweigmann U, Mátyás G, Steinmann B and Stein J I 2006 Different patterns of aortic wall elasticity in patients with Marfan syndrome: a noninvasive follow-up study J. Thorac. Cardiovasc. Surg. 132(4), 811-819.

Boesen M E, Neto M, Souto L A, Pulwicki A, Yerly J, Lebel R M and Frayne R 2015 Fast spin echo imaging of carotid artery dynamics Magn. Reson. Med. 74(4), 1103-1109.

Clough R E, Buerger C, Kolbitsch C, Henningsson M, Taylor P, Prieto C and Schaeffter T 2011 3D aortic motion estimation for image-guided intervention Proc. Int. Soc. Magn. Reson. Med. p. 3740 .

Donati T, Wilson J, Kölbel T and Clough R 2015 Modern diagnostics for type B aortic dissection Gefässchirurgie 20(6), 420-427.

Feinberg D A, Hoenninger J, Crooks L, Kaufman L, Watts J and Arakawa M 1985 Inner volume MR imaging: technical concepts and their application. Radiology 156(3), 743-747.

Ganten M K, Krautter U, von Tengg-Kobligk H, Böckler D, Schumacher H, Stiller W, Delorme S, Kauczor H U, Kauffmann G W and Bock M 2008 Quantification of aortic distensibility in abdominal aortic aneurysm using ECG-gated multi-detector computed tomography Eur. Radiol. 18(5), 966-973.

Goergen C J, Johnson B L, Greve J M, Taylor C A and Zarins C K 2007 Increased anterior abdominal aortic wall motion: possible role in aneurysm pathogenesis and design of endovascular devices J. Endovasc. Ther. 14(4), 574-584.

Hall A J, Busse E F, McCarville D J and Burgess J J 2000 Aortic wall tension as a predictive factor for abdominal aortic aneurysm rupture: improving the selection of patients for abdominal aortic aneurysm repair Ann. Vasc. Surg. 14(2), 152-157.

Hickson S S, Butlin M, Graves M, Taviani V, Avolio A P, McEniery C M and Wilkinson I B 2010 The relationship of age with regional aortic stiffness and diameter JACC Cardiovasc. Imaging 3(12), 1247-1255. 
Hundley W G, Kitzman D W, Morgan T M, Hamilton C A, Darty S N, Stewart K P, Herrington D M, Link K M and Little W C 2001 Cardiac cycle-dependent changes in aortic area and distensibility are reduced in older patients with isolated diastolic heart failure and correlate with exercise intolerance J. Am. Coll. Cardiol. 38(3), 796-802.

Langham M C, Zhou Y, Chirico E N, Magland J F, Sehgal C M, Englund E K, Mohler E R, Guo W, Barhoum S and Wehrli F W 2015 Effects of age and smoking on endothelial function assessed by quantitative cardiovascular magnetic resonance in the peripheral and central vasculature $J$. Cardiovasc. Magn. Reson. 17(1), 1.

Larson A C, White R D, Laub G, McVeigh E R, Li D and Simonetti O P 2004 Self-gated cardiac cine MRI Magn. Reson. Med. 51(1), 93-102.

Li L, Miller K L and Jezzard P 2012 DANTE-prepared pulse trains: A novel approach to motionsensitized and motion-suppressed quantitative magnetic resonance imaging Magn. Reson. Med. 68(5), 1423-1438.

Lin J M, Patterson A J, Chang H C, Gillard J H and Graves M J 2015 An iterative reduced field-of-view reconstruction for periodically rotated overlapping parallel lines with enhanced reconstruction PROPELLER MRI Med. Phys. 42(10), 5757-5767.

Mendes J, Parker D L, Hulet J, Treiman G S and Kim S E 2011 CINE turbo spin echo imaging Magn. Reson. Med. 66(5), 1286-1292.

Morrison T M, Choi G, Zarins C K and Taylor C A 2009 Circumferential and longitudinal cyclic strain of the human thoracic aorta: age-related changes J. Vasc. Surg. 49(4), 1029-1036.

Nelson A J, Worthley S G, Cameron J D, Willoughby S R, Piantadosi C, Carbone A, Dundon B K, Leung M C, Hope S A, Meredith I T et al 2009 Cardiovascular magnetic resonance-derived aortic distensibility: validation and observed regional differences in the elderly J. Hypertens. $\mathbf{2 7}(3), 535-542$.

Nienaber C A, Kische S, Skriabina V and Ince H 2009 Noninvasive imaging approaches to evaluate the patient with known or suspected aortic disease Circ. Cardiovasc. Imaging 2(6), 499-506.

Nienaber C A and Powell J T 2011 Management of acute aortic syndromes Eur. Heart J. p. ehr186.

Ohyama Y, Teixido-Tura G, Ambale-Venkatesh B, Noda C, Chugh A R, Liu C Y, Redheuil A, Stacey R B, Dietz H, Gomes A S et al 2016 Ten-year longitudinal change in aortic stiffness assessed by cardiac MRI in the second half of the human lifespan: the multi-ethnic study of atherosclerosis Eur. Heart J. Cardiovasc. Imaging p. jev332.

Paraskevas K I, Bessias N, Psathas C, Akridas K, Dragios T, Nikitas G, Andrikopoulos V, Mikhailidis D P and Kyriakides Z S 2009 Evaluation of aortic stiffness(aortic pulse-wave velocity) before and after elective abdominal aortic aneurysm repair procedures: a pilot study Open Cardiovasc. Med. J. 3, 173-175.

Pauly J, Le Roux P, Nishimura D and Macovski A 1991 Parameter relations for the Shinnar-Le Roux selective excitation pulse design algorithm IEEE Trans. Med. Imaging 10(1), 53-65.

Redheuil A 2014 Cardiovascular aging: Insights from local and regional measures of aortic stiffness using magnetic resonance imaging Artery Res. 8(2), 66-72.

Sakuragi S and Abhayaratna W P 2010 Arterial stiffness: methods of measurement, physiologic determinants and prediction of cardiovascular outcomes Int. J. Cardiol. 138(2), 112-118. 
Seo J, Choi D, Rienmueller R, Lim J G, Chang Y and Lee J 2009 Suggestion for a new image-based aortic wall stiffness evaluation technique: arterial wall stiffness index Int. J. Cardiovasc. Imaging 25(1), 83-94.

Soljanlahti S, Autti T, Hyttinen L, Vuorio A F, Keto P and Lauerma K 2008 Compliance of the aorta in two diseases affecting vascular elasticity, familial hypercholesterolemia and diabetes: a MRI study Vasc. Health Risk Manag. 4(5), 1103.

Taviani V, Hickson S S, Hardy C J, McEniery C M, Patterson A J, Gillard J H, Wilkinson I B and Graves M J 2011 Age-related changes of regional pulse wave velocity in the descending aorta using Fourier velocity encoded M-mode Magn. Reson. Med. 65(1), 261-268.

Taviani V, Patterson A J, Worters P, Sutcliffe M P, Graves M J and Gillard J H 2010 Accuracy of phase contrast, black-blood, and bright-blood pulse sequences for measuring compliance and distensibility coefficients in a human-tissue mimicking phantom J. Magn. Reson. Imaging 31(1), 160-167.

Tsamis A, Krawiec J T and Vorp D A 2013 Elastin and collagen fibre microstructure of the human aorta in ageing and disease: a review J. R. Soc. Interface 10(83), 20121004.

Van Prehn J, Vincken K, Sprinkhuizen S, Viergever M, Van Keulen J, Van Herwaarden J, Moll F and Bartels L 2009 Aortic pulsatile distention in young healthy volunteers is asymmetric: analysis with ECG-gated MRI Eur. J. Vasc. Endovasc. Surg. 37(2), 168-174.

Voges I, Jerosch-Herold M, Hedderich J, Pardun E, Hart C, Gabbert D D, Hansen J H, Petko C, Kramer H H and Rickers C 2012 Normal values of aortic dimensions, distensibility, and pulse wave velocity in children and young adults: a cross-sectional study J. Cardiovasc. Magn. Reson. 14(1), 1.

Wargo C J, Moore J and Gore J C 2013 A comparison and evaluation of reduced-FOV methods for multi-slice 7T human imaging Magnetic Resonance Imaging 31(8), 1349-1359.

Zho S Y, Park J, Choi J Y and Kim D H 2010 Respiratory motion compensated MR cholangiopancreatography at 3.0 Tesla J. Magn. Reson. Imaging 32(3), 726-732. 